\title{
Dorsal Morphology of the Calcaneus-An Osteological Study
}

\author{
Rebecca Murerwa, Thomas Amuti, Nikita Muuthuri, Anita Wambui, Innocent Ouko, Julius Ogeng'o \\ Department of Human Anatomy, University of Nairobi, Kenya
}

Correspondence to: Thomas Amuti; email: tomamuti@gmail.com.

\begin{abstract}
Background: The calcaneus exhibits variations in articular facets and calcaneal sulcus width. These variations influence occurrence of osteoarthritic conditions, whose prevalence is significant in our setting, and are essential during surgeries involving the foot. Despite this, local data on calcaneal facets remain scarce. Methods: Sixty calcanei were obtained from the National Museum of Kenya. The number, type and shapes of their facets were documented and calcaneal sulcus width measured. Images were taken and collected data were represented in tables and figures. Results: The calcanei had 1-3 articular facets. The common calcanei type noted was type $1 \mathrm{~B}$ on the right calcaneus and $1 \mathrm{~B}$ on the left. The least was type 4 on the right and $2 \mathrm{C}$ on the left. In terms of shape, types 1 and 4 calcanei had non-rounded facets while type 2 had rounded facets. The width of the sulcus calcanei was narrowest among type 1 calcanei in the right foot at $0.53 \mathrm{~cm}$. On the left foot, type 2 calcanei
\end{abstract}

\section{Introduction}

The calcaneus is the largest tarsal bone (1-3). It varies in the number and shape of talo-calcaneal facets. The bone is known to have one to three facets. Morphologically, these facets have further been classified into types. Type 1 calcanei have two facets: anteromedial and posterior. Type 2 has three facets: anterior, middle and posterior. Type 3 have two facets also-middle and posterior, while type 4 have one facet - a combined anteroposteromedial facet. Population variations in the prevalence of the types of calcanei have been noted. Data from Caucasian $(4,5)$ populations show that their calcanei predominantly have three facets. In Indian (6), Nigerian (4), Thai (7) and Turkish populations, two facets per calcanei were most common (8).

Regarding its shape, a study done on the Nigerian population (1) showed that their anterior and middle facets were mostly oval, the anteromedial facet was elongated and the posterior facet was rounded. Notable variations on the calcaneal sulcus have been reported. The calcaneal sulcus is the dorsal groove that articulates with the tarsal sulcus to form the sinus tarsi at the subtalar joint. Since the tarsi allows for the passage of had narrower $(0.455 \mathrm{~cm}$, range $0.35-0.60)$ calcaneal sulcus width. Conclusion: Our findings may aid in surgeries of the foot and in providing a link between local calcaneal variations and prevalence of osteoarthritic foot conditions.

Keywords: Calcaneal-facets, Sinus tarsi, Arthritis

Ann Afr Surg. 2020;17(3):112-115

DOI: http://dx.doi.org/10.4314/aas.v17i3.5

Conflicts of Interest: None

Funding: None

(C) 2020 Author. This work is licensed under the Creative

Commons Attribution 4.0 International License

Submitted: 13 September 2019

Revised: 17 January 2020

Accepted: 18 February 2020

Online first: 29 May 2020

structures, its width is of significant clinical importance. Collected data show variations in this width with the Turkish (7), Indian (10) and Korean populations (11) having values of $6.15,21.1$ and $5.16 \mathrm{~mm}$ respectively. Variations in width size could have clinical implications such as bone and tendon pain by compressing structures.

The reported variations of the calcanei, especially in the type as well as shape of the facets, have been shown to influence surgical procedures (11). Some disorders such as pes planus $(12,13)$ have been shown to be significantly prevalent (43.2\%) in Kenya (14). However, data are scarce on the morphological variations of the calcaneal facets and calcaneal sulcus in our setting. This data may be relevant in contributing to the pre-existing scanty knowledge on the influence of variations in the talocalcaneal facets in our setting, as well as providing local data that may be useful in orthopaedic surgeries.

\section{Materials and Methods}

The present study is a descriptive cross-sectional study, with a random sample of 30 paired dried calcanei from The National Museum of Kenya in Nairobi. The specimens used were of adult Kenyans (18-65 years). Stratification by gender was not possible due to missing 
information from the identification cards. Calcanei that showed structural deformities were also not used for the study. Approval for the use of osteological specimens was provided by the Department of Human Anatomy, University of Nairobi, and the National Museum of Kenya.

The calcanei were classified based on the calcaneal facet number and type, following the classification by Laxmi et al. (2017) (15):

Type 1: One continuous facet is situated on the sustentaculum tali extending to the antero-medial corner of the distal part of the calcaneus. The facet can either be constricted or not constricted. The other facet is posterior to the first. The constriction of the anterior facet and the posterior is defined as a temporary point of separation of the two facets by Jung et al. (2015) (11), and the extent of the constriction/separation is defined as the degree of separation. The degree of separation of the facets ranges from 2.00 to 1.00 , with a grade of 2.00 being facets whose anterior and middle facets are completely separated (type 2 facets), 1.00 as those that are fused into a single facet (with no point of constriction), and finally, facets with constrictions being graded between 1.00 and 2.00. Specifically, the degree of separation in constricted facets is calculated using the following formula: $1.00+$ the ratio of disconnected parts at the constriction to the total width of the anterior and middle facets.

Type 2: Three articular facets are present, one on the sustentaculum tali (middle facet), one anterior facet and the other on the distal part of the calcaneus (posterior facet). The distance between the middle and anterior facets of the sustentaculum facets further subdivide the type 2 facets into four subdivisions: type $2 \mathrm{~A}$ has the distance between the anterior and middle facets being $<2$ $\mathrm{mm}$; $2 \mathrm{~B}$ between $2-5 \mathrm{~mm} ; 2 \mathrm{C}>5 \mathrm{~mm}$ with a large anterior facet; and 2D >5 mm with a small anterior facet. Type 3: Two facets are present with the anterior one restricted to the sustentaculum tali.

Type 4: A single facet is situated on the sustentaculum tali extending to the antero-medial corner of the distal part of the body of the calcaneus. This facet is continuous with the posterior talar facet of the calcaneus.

The shapes of each facet per calcanei were also documented. A vernier calipers was used to measure the width of the calcaneal sulcus. The width of the sulcus tarsi was defined as the shortest distance (16) between the antero-medial facet and the posterior facet in type 1, the

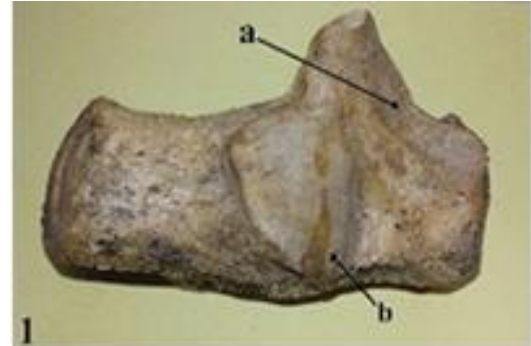

Figure 1. Type 1 facet a. anteromedial facets (non-constricted), b. posterior facet

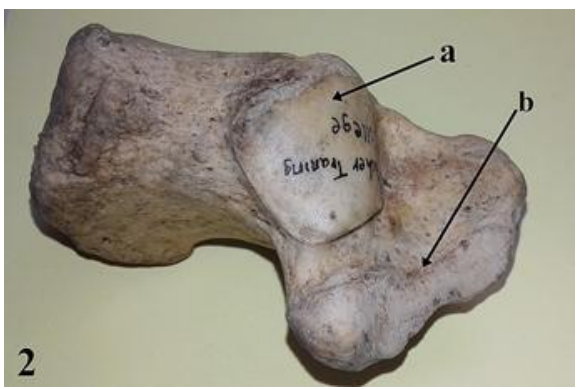

Figure 2. Type 1 facet: $\mathrm{a}$ - posterior facet, $\mathrm{b}$ - anteromedial facets (constricted)

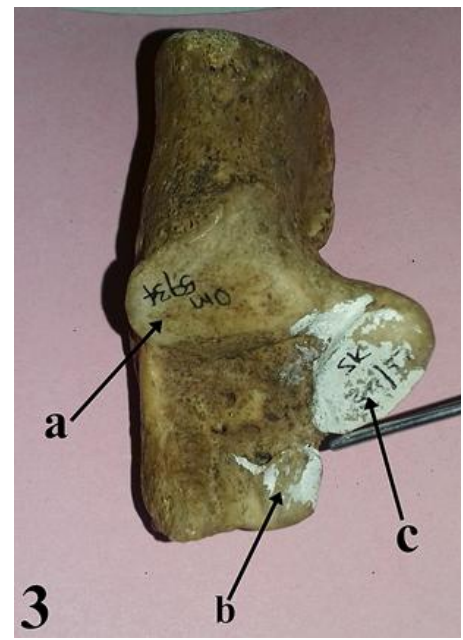

Figure 3. Type 2 facet: $\mathrm{a}$ - posterior facet, $\mathrm{b}$ - anterior facet, $\mathrm{c}-$ middle facet

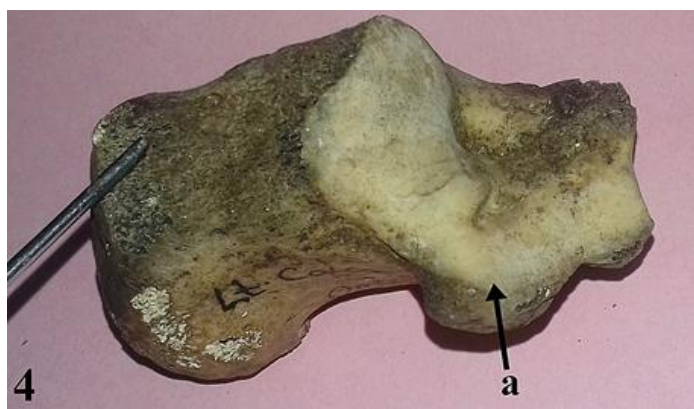

Figure 4. Type 4 facet: a - combined antero-medio-posterior facet

shortest distance between the middle and posterior facets in type 2 , and the shortest distance between the middle and posterior facets in type 3. At least three measurements of the calcaneal sulcus were taken and the average of these taken as the width of the calcaneal sulcus. Data collected were entered into and analyzed 
using SPSS version 21. $\mathrm{p}$ value was considered significant at $95 \%$ confidence interval. The findings are represented in tables and figures.

\section{Results \\ Morphology of the calcaneal facets}

All the calcanei had articular facets that ranged in number from 1 to 3 . The most prevalent calcaneus type noted on the right was type $1 \mathrm{~B}$ while type 4 was the least prevalent. On the left, type $1 \mathrm{~B}$ was most common while types $2 \mathrm{C}$ and 4 were the least common (Table 1). Paired $t$ test revealed significant differences between the right and left calcaneal types $(p=0.001)$. Type 3 calcaneus was not documented in this study.

All combined anterior and middle facets (type 1) and the combined single facet (type 4) calcanei were nonrounded, type 1 calcanei were elongated and type $4 \mathrm{v}$ shaped. Calcanei having the type 2 facet configuration (all three facets) had rounded anterior, middle and posterior facets. These findings are similar on both the right and left calcaneal groups. Paired $t$ test revealed no significant differences between right and left calcaneal shapes.

Table 1. Frequency (\%) of various types of calcaneal facets

\begin{tabular}{lllllllll} 
VARIABLE & $1 \mathrm{~A}$ & $1 \mathrm{~B}$ & $2 \mathrm{~A}$ & $2 \mathrm{~B}$ & $2 \mathrm{C}$ & $2 \mathrm{D}$ & 3 & 4 \\
\hline $\begin{array}{l}\text { RIGHT } \\
\text { CALCANEAL }\end{array}$ & 20 & 47 & 13 & 10 & 6 & 0 & 0 & 4 \\
TYPE & & & & & & & & \\
\hline $\begin{array}{l}\text { LEFT } \\
\text { CALCANEAL }\end{array}$ & 22 & 40 & 13 & 13 & 4 & 0 & 0 & 8 \\
TYPE & & & & & & & & \\
\hline
\end{tabular}

\section{Width of the calcaneal facets}

The width of the sulcus calcanei was narrowest among calcaneal with a type 1 facet arrangement in the right foot at $0.53 \mathrm{~cm}(0.30-1.20)$. On the left foot however, calcanei with a type 2 arrangement had the narrower calcaneal sulcus width of $0.455 \mathrm{~cm}(0.35-0.60)$ (Table 2).

Table 2. Width of the sulcus calcanei by type

\begin{tabular}{llll}
\hline CALCANEUS & $\begin{array}{l}\text { CALCANEAL } \\
\text { TYPE }\end{array}$ & $\begin{array}{l}\text { MEAN WIDTH OF } \\
\text { CALCANEAL } \\
\text { SULCUS }(\mathrm{cm})\end{array}$ & $\begin{array}{l}\text { RANGE } \\
(\mathrm{cm})\end{array}$ \\
\hline RIGHT & 1 & 0.53 & $0.30-1.20$ \\
\cline { 2 - 4 } CALCANEUS & 2 & 0.56 & $0.34-1.49$ \\
\hline LEFT & 1 & 0.46 & $0.30-0.78$ \\
\cline { 2 - 4 } CALCANEUS & 2 & 0.455 & $0.35-0.60$ \\
\hline
\end{tabular}

\section{Discussion}

\section{Predominant calcaneal facet type 1}

The calcaneal facet type 1 was the most dominant in the study setting. Our findings differed from those of the British (4) and American (5) populations, where the type 2 was the predominant calcanei facet type. Our findings were however similar to those of the Indian (6), Nigerian (4), Thai (7) and Turkish populations (8) with type 1 most prevalent. The variance could be due to genetic heterogeneity where, owing to differences in genetic makeup, populations might bear differences in calcaneal anatomy similar to those seen in other bones (17).

The facet number is an important factor in subtalar joint stability and formation of osteophytes in osteoarthritis. Studies have shown that subtalar joints that have the type 2 facet configuration are comparatively more stable and have a lower chance of developing arthritis (12). This is likely because the facets with the anterior, middle and posterior facets (type 2) provide an 'osseous tripod' for the talus to sit on and to prevent excess motion of the talar head. Thus, the subtalar joint with this tripod support is less likely to suffer trauma or biomechanical stress, and the incidence of osteoarthritis has been shown to be lower in such cases (11). Second, facets with a type 1 configuration have smaller facet areas than the rest. Therefore, in these individuals, more pressure may be exerted on these facets, leading to damage of the facet tissue and subsequent osteophyte formation (11). It is also important to note that individuals with types 1 and 2 facets have been shown to have asymmetric wear patterns which might cause heavy pain, possibly leading to a habitually inclined position of the foot during walking that predisposes to osteophyte formation and subsequent osteoarthritis (11).

\section{Calcaneal facet shape}

The most common shape among the combined anterior and middle facets (type 1) and the combined single facet (type 4) calcanei was non-rounded. Further, these combined anterior and middle facets were elongated whereas the combined single facets were $\mathrm{v}$-shaped. Calcanei having the type 2 facet configuration on the other hand had rounded anterior, middle and posterior facets. These findings were similar on both the right and left calcaneal groups and to data derived from the Nigerian population (1).

Rounded facets compared with non-rounded facets have been shown to have larger surface areas and as such may offer greater stability than the latter (16). Owing to the significant number of cases of osteoarthritic conditions and paucity of data on the morphological variations in the number of calcaneal facets in our setting, findings of this study may suggest that the of presence non-rounded 
facets may add to the major factors that contribute to the high number of osteoarthritic conditions in our setting.

\section{Calcaneal sulcus width}

The calcaneal sulcus in our setting was similar in width to those reported from the Korean (11) and Anatolian populations (17) where the average values were 5.16 and $5.98 \mathrm{~mm}$ respectively. Our values were however lower than those reported in the Indian (9) and Turkish (7) populations whose the values were 21.1 and $6.15 \mathrm{~mm}$ respectively. The population differences may be due to genetic heterogeneity among populations. In our setting therefore, it may be important for surgeons to note the different values especially during subtalar implants, flaps and foot prostheses to avoid post-surgical pain, and in any surgeries involving the foot.

\section{Conclusion}

Data obtained in our setting varies with that from other populations. These data may prove useful in surgical procedures of the foot as well as in providing a possible basis on the influence of calcaneal facet variation in the local prevalence of osteoarthritis.

\section{References}

1. Ukoha U, Obazie IF, Chioma O, et al. Study of the morphologic and morphometric patterns of talar articular facets on dry adult calcaneal bones in South-Eastern Nigerian population. Revista Argentina de Anatomía. 2017;9(2):58-66.

2. Ilhan OT, Tetiker $\mathrm{H}$, Tastemur $\mathrm{Y}$, et al. Morphometric measurements of calcaneus: Boehler's angle and bone length estimation. Cumhuriyet Universitesi Fen-Edebiyat Fakültesi Fen Bilimleri Dergisi. 2017;38(2):256-263.

3. Loudon J, Manske R, Reiman M, et al. Clinical mechanics and kinesiology. Leeds 2013: Human Kinetics.

4. Alpay M, Yegin B, Yucel F, et al. A case study for the anthropometric measurement of calcaneus. Osmangazi $\mathrm{J}$ of Med. 2018;40(2):6-12.

5. Nemade K, Meshram M, Kasote A, et al. Arthritis of the subtalar joint associated with sustentaculum tali facet configuration. Int J Anat Res. 2014;2(4):684-88.
6. Garg R, Dagal N, Kumar S, et al. Study of patterns of talar articular facets of human calcanei and their clinical implications in population of Rajasthan. Indian J Basic Appl Med Res. 2013;7(2):643-650.

7. Uygur M, Atamaz F, Celik S, et al. The types of talar articular facets and morphometric measurements of the human calcaneus bone in Turkish race. Arch Orthop Trauma Surg. 2009; 129:909-14.

8. Sitthichai I, Nongnut U, Porntip B, et al. Types of facets on the superior articular surface of Isan-Thai dried calcanei. Int $\mathbf{J}$ Morphol. 2015;33(4):1549-52.

9. Agarwal S, Garg S, Vasudeva N, et al. Subtalar joint instability and calcaneal spurs associated with configuration of the articular facets of adult human calcaneum in the Indian population. J Clin Diagn Res. 2016;10(9):AC05-AC09

10. Sarvaiya BJ, Patel SV, Single G, et al. The types of talar articular facets and morphometric measurements of the human calcaneum bone of Gujarat Region. Nat. J Integr Res Med. 2012;3:34-8.

11. Jung MH, Choi BY, Lee JY, et al. Types of subtalar joint facets. Surg Radiol Anat. 2015;37(6):629-38.

12. Yang Y, Cheng HW, Xiong ZR, et al. Classification and morphological parameters of the calcaneal talar facet: Which type is more likely to cause osteoarthritis in Chinese population. Biomed Res Int. 2019.

13. Muthukumaravel N, Ravichandran D, Melani RS. Human calcaneal facets for the talus: Patterns and clinical implications. J Clin Diagn Res. 2011;5:791-94.

14. Greer RE. Pes Planus. In: S Terry Canale. Campbell's operative prthopaedics. 9th ed. St. Louis: Mosby - Year Book 1988:1720-25.

15. Laxmi V, Ritu M, Ravikant S, et al. A morphological and morphometric study of human calcanei and their articular facets. Ann Geriatr Med Res. 2018;5(1):28-32.

16. Jagdev SK, Anterpreet KA, Navprateek SK, et al. Morphology of talar articular facets of calcaneus and its clinical implications. Kashmir J Med Sci. 2015;1(1).

17. Wallace I, Middleton K, Lublinsky S, et al. Functional significance of genetic variation underlying limb bone diaphyseal structure. Am J Phys Anthropol. 2010;143(1):2130. 